\title{
Prediction for Progressively Type-II Censored Competing Risks Data from the Half-Logistic Distribution
}

\author{
Essam K. AL-Hussaini ${ }^{1}$, Alaa H. Abdel-Hamid ${ }^{2, \star}$, Atef F. Hashem ${ }^{2}$ \\ ${ }^{1}$ Department of Mathematics, Faculty of Science, Alexandria University, Egypt \\ ${ }^{2}$ Mathematics and Computer Science Department, Faculty of Science, Beni-Suef University, Egypt
}

\section{ARTICLE INFO}

\section{Article History}

Received 13 Apr 2018

Accepted 21 Dec 2019

\section{Keywords}

Maximum likelihood predictor Bayesian prediction

Competing risks model Progressive type-II censoring

Half-logistic distribution

Two-sample prediction

Simulation

2000 Mathematics Subject Classification: $62 \mathrm{~N} 01,62 \mathrm{M} 20$, 60G25.

\section{ABSTRACT}

Point and interval predictions of the s-th order statistic in a future sample are discussed. The informative sample is assumed to be drawn from a general class of distributions which includes, among others, Weibull, compound Weibull, Pareto, Gompertz and half-logistic distributions. The informative and future samples are progressively type-II censored, under competing risks model, and assumed to be obtained from the same population. A special attention is paid to the half-logistic distribution. Using six different progressive censoring schemes, numerical computations are carried out to illustrate the performance of the procedure. An illustrative example based on real data is also considered. The biases, mean squared prediction errors of the maximum likelihood predictors, coverage probabilities and average interval lengths of the Bayesian prediction intervals are computed via a simulation study.

(C) 2020 The Authors. Published by Atlantis Press SARL. This is an open access article distributed under the CC BY-NC 4.0 license (http://creativecommons.org/licenses/by-nc/4.0/).

\section{INTRODUCTION}

Prediction is an important problem in the statistical inference. It is the problem of inferring the values of unknown observables (future observations), or functions of such observables, from current available (informative) observations. One-sample and two-sample schemes are two commonly used schemes of prediction. A predictor could be a point or an interval predictor. Prediction has many applications in the field of quality control, reliability, medical sciences, business, engineering and has been studied by many authors, including [1-9]. AL-Hussaini et al. [10] obtained Bayesian one-sample prediction of future order statistics from the half-logistic distributions, based on progressively type-II censored sample under competing risks model.

In reliability and survival analysis, engineering, demographic, actuarial literature, econometric, biological or medical studies, the units might fail owing to one of various causes. These causes compete in order to fail the units. This is called in the statistical literature "competing risks." The data for the competing risks model consist of the lifetime of the failed unit and an indicator variable denoting the cause of failure. In this article, we study the competing risks model under the assumption of only two independent causes of failure and the lifetimes of $n$ units to be tested are independent and identically distributed (iid). We assume that, for $i=1, \ldots, n, T_{i 1}$ and $T_{i 2}$ are two iid random variables (RVs) and $T_{i}=\min \left\{T_{i 1}, T_{i 2}\right\}$ where $T_{i j}$ denotes the lifetime of unit $i$ when it fails because of cause $j, j=1,2$.

Let, for $j=1,2$, the cumulative distribution function (CDF), $F_{j}(t)$, of a $\mathrm{RV} T_{i j}$ be of the form

$$
F_{j}(t) \equiv F_{j}\left(t ; \beta_{j}\right)=\left\{\begin{array}{cc}
1-\exp \left[-u_{j}(t)\right], & 0 \leq b_{1} \leq t \leq b_{2} \leq \infty \\
0, & \text { otherwise }
\end{array}\right.
$$

where $\beta_{j}$ is a positive parameter and $u_{j}(t) \equiv u_{j}\left(t ; \beta_{j}\right)=-\ln \left[1-F_{j}(t)\right]$ is a non-negative, continuous, monotone increasing and differentiable function of $t$ such that $u_{j}(t) \rightarrow 0$, as $t \rightarrow b_{1}^{+}$and $u_{j}(t) \rightarrow \infty$, as $t \rightarrow b_{2}^{-}, j=1,2$. In particular, any CDF with positive domain (suitable for life testing) is a special case of (1). For example, the Weibull, exponential, Rayleigh, compound Weibull (Burr type XII), compound exponential (Lomax), compound Rayleigh, Pareto, beta, Gompertz and compound Gompertz distributions are all special cases of (1).

${ }^{*}$ Corresponding author. Email: hamid_alh@science.bsu.edu.eg 
See $[1,11,12]$. It may be remarked that distributions, defined over the whole real line may be represented by (1) with the appropriate choice of $u_{j}(t)$. The probability density function (PDF), $f_{j}(t)$, corresponding to CDF $(1)$, is given by

$$
f_{j}(t) \equiv f_{j}\left(t ; \beta_{j}\right)=u_{j}^{\prime}(t) \exp \left[-u_{j}(t)\right]
$$

where $u_{j}^{\prime}(t)$ is the hazard rate function (HRF) and exp $\left[-u_{j}(t)\right]$ is the survival function. The prime denotes derivative with respect to $t$, throughout.

The CDF of $T_{i}$ is given by

$$
\begin{aligned}
F(t) \equiv F(t ; \boldsymbol{\beta}) & =1-\prod_{j=1}^{2}\left[1-F_{j}(t)\right] \\
& =1-\exp [-u(t)],
\end{aligned}
$$

where

$$
u(t) \equiv u(t ; \boldsymbol{\beta})=\sum_{j=1}^{2} u_{j}(t)
$$

and $\boldsymbol{\beta}=\left(\beta_{1}, \beta_{2}\right)$ is a vector of positive parameters.

The $\operatorname{PDF} f(t)$, corresponding to $\operatorname{CDF}(3)$, is given by

$$
f(t) \equiv f(t ; \boldsymbol{\beta})=u^{\prime}(t) \exp [-u(t)]
$$

In medical or industrial applications, censoring usually applies when the experimenter is unable to get total information on lifetimes for each unit or reducing the total test time and the associated cost. Type-I and type-II are two commonly used censoring schemes (CSs), see, for example, $[13,14,15]$. These types of censoring cannot allow the experimenter to remove units from a life test at various stages during the experiment. The experimenter can overcome this problem by using progressive type-II censoring which is considered to be a generalization of type-II censoring. It allows the experimenter to remove units from a life test at various stages during the experiment. For more details on progressive censoring, see [16,17].

In this paper, based on two-sample prediction technique, we discuss point and interval predictions of the s-th order statistic in a future sample based on progressively type-II censored sample generated from a general class of distributions under competing risks model. The results are then applied to the half-logistic population.

The rest of the article is organized as follows: In Section 2, progressive type-II censoring is described. In Section 3, the maximum likelihood predictors (MLPs) are provided. Section 4 presents the Bayesian two-sample prediction method. The half-logistic distribution is considered in Section 5. In Section 6, numerical computations and simulations are given. Concluding remarks are finally presented in Section 7.

\section{PROGRESSIVE TYPE-II CENSORING}

The progressive type-II censoring under competing risks model can be applied as follows:

1. Suppose that $n$ units are put on the experiment.

2. Suppose $m(<n)$ and CS $R_{1}, \ldots, R_{m}$ are fixed before the experiment and $n=m+\sum_{i=1}^{m} R_{i}$, where $R_{i}$ represents the number of removed units at the $i$-th failure, $i=1, \ldots, m$.

3. The experiment terminates when the $m$-th failure occurs at which $R_{m}=n-m-\sum_{i=1}^{m-1} R_{i}$ surviving units are all removed from the experiment.

4. The data from progressively type-II censored sample under competing risks model are as follows: $\left(t_{1: m: n} ; a_{1} ; R_{1}\right)<\ldots<$ $\left(t_{m: m: n} ; a_{m} ; R_{m}\right)$ where $a_{1}, \ldots, a_{m}$ denote the $m$ causes of failure.

Note that the complete sample case is achieved when $R_{i}=0, i=1, \ldots, m$ and $n=m$. But, if $R_{i}=0, i=1, \ldots, m-1$ and $R_{m}=n-m$, then progressive type-II CS reduces to traditional type-II CS. 
Based on Equations (1) and (2), and the progressively type-II censored sample, the likelihood function is then given by, see [18],

$$
\begin{aligned}
L(\boldsymbol{\beta} ; \mathbf{t}) & \propto \prod_{i=1}^{m}\left[\prod_{j=1}^{2}\left(h_{j}\left(t_{i}\right)\right)^{I\left(a_{i}=j\right)}\left(\prod_{l=1}^{2}\left(1-F_{l}\left(t_{i}\right)\right)\right)^{R_{i}+1}\right] \\
& =\prod_{j=1}^{2} \prod_{r=1}^{m_{j}}\left[u_{j}^{\prime}\left(t_{r j}\right)\right] \exp \left[-\sum_{i=1}^{m}\left(R_{i}+1\right) u\left(t_{i}\right)\right],
\end{aligned}
$$

where $h_{j}\left(t_{i}\right)=f_{j}\left(t_{i}\right) /\left(1-F_{j}\left(t_{i}\right)\right)$ is the HRF, $t_{i}=\min \left\{t_{i 1}, t_{i 2}\right\}, i=1, \ldots, m, \mathbf{t}=\left(t_{1}, \ldots, t_{m}\right), m_{j}=\sum_{i=1}^{m} I\left(a_{i}=j\right)$ is the number of failures due to cause $j, j=1,2, m=m_{1}+m_{2}$ and

$$
I\left(A_{i}=j\right)= \begin{cases}1, & A_{i}=j \\ 0, & \text { otherwise }\end{cases}
$$

and $A_{i}$ is a discrete RV with realization $a_{i}$.

\section{MAXIMUM LIKELIHOOD PREDICTOR}

Suppose that $\left(T_{1: m: n} ; a_{1} ; R_{1}\right), \ldots,\left(T_{m: m: n} ; a_{m} ; R_{m}\right)$ is an informative progressively type-II censored sample of size $m$ obtained from a sample of size $n$ with progressive CS $\left(R_{1}, \ldots, R_{m}\right)$ under competing risks model. Suppose also that $\left(Y_{1: m^{\star}: n^{\star}} ; a_{1}^{\star} ; R_{1}^{\star}\right), \ldots,\left(Y_{m^{\star}}: m^{\star}: n^{\star} ; a_{m^{\star}}^{\star} ; R_{m^{\star}}^{\star}\right)$ is a future (unobserved) independent progressively type-II censored sample of size $m^{\star}$ obtained from a sample of size $n^{\star}$ with progressive CS $\left(R_{1}^{\star}, \ldots, R_{m^{\star}}^{\star}\right)$ under competing risks model, where $a_{1}^{\star}, \ldots, a_{m^{\star}}^{\star}$ denote the $m^{\star}$ causes of failure. Our object, in this section, is to obtain MLP of the $s$-th order statistic, $Y_{s}$, in the future sample, $s=1, \ldots, m^{\star}$, based on an informative progressively type-II censored sample.

Following Balakrishnan et al. [19] and Kamps and Cramer [20], the conditional PDF of $Y_{s}, s=1, \ldots, m^{\star}$ is given by

$$
g\left(y_{s} \mid \boldsymbol{\beta}\right)=B_{s-1}^{\star} f\left(y_{s}\right) \sum_{\ell=1}^{s} c_{\ell}\left[1-F\left(y_{s}\right)\right]^{\gamma_{\ell}-1},
$$

where

$$
\begin{aligned}
B_{s-1}^{\star} & =\prod_{\ell=1}^{s} \gamma_{\ell}, \\
\gamma_{\ell} & =n^{\star}-\sum_{q=1}^{\ell-1}\left(R_{q}^{\star}+1\right), \quad\left(\text { if } \ell=1, \gamma_{1}=n^{\star}\right), \\
c_{\ell} & =\prod_{d=1(d \neq \ell)}^{s} \frac{1}{\gamma_{d}-\gamma_{\ell}}, \quad\left(\text { if } s=1, c_{1}=1\right) .
\end{aligned}
$$

The conditional PDF (7) of $Y_{s}$, using Equations (3) and (5), becomes

$$
g\left(y_{s} \mid \boldsymbol{\beta}\right)=B_{s-1}^{\star} u^{\prime}\left(y_{s}\right) \sum_{\ell=1}^{s} c_{\ell} \exp \left[-\gamma_{\ell} u\left(y_{s}\right)\right] .
$$

The predictive likelihood function (PLF) of $Y_{s}$ and $\boldsymbol{\beta}$ is given by, see, for example, [5],

$$
\begin{aligned}
g^{\star}\left(y_{s}, \boldsymbol{\beta} ; \mathbf{t}\right) & =g\left(y_{s} \mid \boldsymbol{\beta}\right) L(\boldsymbol{\beta} ; \mathbf{t}) \\
& =B_{s-1}^{\star} u^{\prime}\left(y_{s}\right) \sum_{\ell=1}^{s} c_{\ell} \exp \left[-\gamma_{\ell} u\left(y_{s}\right)\right] \times \prod_{j=1}^{2} \prod_{r=1}^{m_{j}}\left[u_{j}^{\prime}\left(t_{r j}\right)\right] \exp \left[-\sum_{i=1}^{m}\left(R_{i}+1\right) u\left(t_{i}\right)\right] .
\end{aligned}
$$

The logarithm of PLF (9) is then given by

$$
\ln g^{\star}=\ln B_{s-1}^{\star}+\ln u^{\prime}\left(y_{s}\right)+\ln \sum_{\ell=1}^{s} c_{\ell} \exp \left[-\gamma_{\ell} u\left(y_{s}\right)\right]+\sum_{j=1}^{2} \sum_{r=1}^{m_{j}} \ln u_{j}^{\prime}\left(t_{r j}\right)-\sum_{i=1}^{m}\left(R_{i}+1\right) u\left(t_{i}\right) .
$$

The predictive maximum likelihood estimates (PMLEs) $\left(\widehat{\beta_{1}} \widehat{\beta_{2}}\right)$ of $\left(\beta_{1}, \beta_{2}\right)$ and MLP $\widehat{y_{s}}$ of $y_{s}$ can be obtained by setting to zero the first partial derivatives of (10) with respect to $\left(\beta_{1}, \beta_{2}\right)$ and $y_{s}$ and then solve the resultant equations using any iteration method, the predictors yield. 
A $\left(1-\alpha^{\star}\right) 100 \%$ prediction intervals (PIs) for future order statistic $Y_{s}$ can be constructed, using the MLP $\widehat{y}_{s}$, as follows

$$
\left(\widehat{y}_{s}-z_{\alpha^{\star} / 2} \sqrt{\operatorname{var}\left(\hat{y}_{s}\right)}, \widehat{y}_{s}+z_{\alpha^{\star} / 2} \sqrt{\operatorname{var}\left(\hat{y}_{s}\right)}\right),
$$

where $z_{\alpha^{\star} / 2}$ is the percentile of standard normal distribution with right-tale probability of $\alpha^{\star} / 2$, $\operatorname{var}\left(\widehat{y}_{s}\right)$ can be calculated using the inverse of observed Fisher information matrix, see, for example, [21,22].

\section{BAYESIAN TWO-SAMPLE PREDICTION}

In the following section, we discuss the Bayesian predictive intervals for future order statistics under progressively type-II censored competing risks data. Suppose that both of the two parameters $\beta_{1}$ and $\beta_{2}$ are unknown. Suppose also that the prior belief of the experimenter is that they are independent and each of them has a gamma distribution. Therefore, the joint prior density for the parameters is of the form

$$
\pi\left(\beta_{1}, \beta_{2}\right)=\pi_{1}\left(\beta_{1}\right) \pi_{2}\left(\beta_{2}\right),
$$

where

$$
\begin{aligned}
& \pi_{1}\left(\beta_{1}\right)=\frac{\alpha_{2}^{\alpha_{1}}}{\Gamma\left(\alpha_{1}\right)} \beta_{1}^{\alpha_{1}-1} \exp \left[-\alpha_{2} \beta_{1}\right], \quad \beta_{1}>0, \quad\left(\alpha_{1}, \alpha_{2}>0\right), \\
& \pi_{2}\left(\beta_{2}\right)=\frac{\alpha_{4}^{\alpha_{3}}}{\Gamma\left(\alpha_{3}\right)} \beta_{2}^{\alpha_{3}-1} \exp \left[-\alpha_{4} \beta_{2}\right], \quad \beta_{2}>0, \quad\left(\alpha_{3}, \alpha_{4}>0\right) .
\end{aligned}
$$

Waller and Waterman [23] showed that the family of gamma distribution may be used as priors in Bayesian reliability analysis. Thus, gamma prior density may be rich enough to cover the prior belief of the experimenter.

From (12) and (13), Equation (11) becomes

$$
\pi\left(\beta_{1}, \beta_{2}\right)=D \beta_{1}^{\alpha_{1}-1} \beta_{2}^{\alpha_{3}-1} \exp \left[-\left(\alpha_{2} \beta_{1}+\alpha_{4} \beta_{2}\right)\right], \quad \beta_{1}, \beta_{2}>0, \quad\left(\alpha_{1}, \alpha_{2}, \alpha_{3}, \alpha_{4}\right)>0,
$$

where $D=\alpha_{2}^{\alpha_{1}} \alpha_{4}^{\alpha_{3}} /\left(\Gamma\left(\alpha_{1}\right) \Gamma\left(\alpha_{3}\right)\right)$.

From (6) and (14), the joint posterior PDF of $\beta_{1}$ and $\beta_{2}$ is then given by

$$
\pi^{*}\left(\beta_{1}, \beta_{2} \mid \mathbf{t}\right)=K^{-1} \eta \exp [-\delta], \quad\left(\beta_{1}, \beta_{2}\right)>0,
$$

where

$$
\begin{aligned}
& K=\int_{0}^{\infty} \int_{0}^{\infty} \eta \exp [-\delta] \mathrm{d} \beta_{1} \mathrm{~d} \beta_{2}, \\
& \eta=\beta_{1}^{\alpha_{1}-1} \beta_{2}^{\alpha_{3}-1} \prod_{j=1}^{2} \prod_{r=1}^{m_{j}} u_{j}^{\prime}\left(t_{r j}\right), \\
& \delta=\alpha_{2} \beta_{1}+\alpha_{4} \beta_{2}+\sum_{i=1}^{m}\left(R_{i}+1\right) u\left(t_{i}\right) .
\end{aligned}
$$

Using Equations (8) and (15), the Bayesian predictive PDF of $Y_{s}, s=1, \ldots, m^{\star}$, is then given by

$$
\begin{aligned}
f^{\star}\left(y_{s} \mid \mathbf{t}\right) & =\int_{0}^{\infty} \int_{0}^{\infty} g\left(y_{s} \mid \beta_{1}, \beta_{2}\right) \pi^{*}\left(\beta_{1}, \beta_{2} \mid \mathbf{t}\right) \mathrm{d} \beta_{1} \mathrm{~d} \beta_{2} \\
& =K^{-1} B_{s-1}^{\star} \sum_{\ell=1}^{s} c_{\ell} \int_{0}^{\infty} \int_{0}^{\infty} \eta u^{\prime}\left(y_{s}\right) \exp \left[-\left(\delta_{2}+\gamma_{\ell} u\left(y_{s}\right)\right)\right] \mathrm{d} \beta_{1} \mathrm{~d} \beta_{2} .
\end{aligned}
$$

The Bayesian prediction intervals (BPIs) for $Y_{s}, s=1, \ldots, m^{\star}$, are obtained by evaluating $P\left(Y_{s}>v \mid \mathbf{t}\right)$ for some given values of $v$. It follows from (16) that

$$
P\left(Y_{s}>v \mid \mathbf{t}\right)=K^{-1} B_{s-1}^{\star} S(v), \quad v>0
$$

where

$$
S(v)=\sum_{\ell=1}^{s} \frac{c_{\ell}}{\gamma_{\ell}} \int_{0}^{\infty} \int_{0}^{\infty} \eta \exp \left[-\left(\delta+\gamma_{\ell} u(v)\right)\right] \mathrm{d} \beta_{1} \mathrm{~d} \beta_{2} .
$$


Since $1=P\left(Y_{s}>0 \mid \mathbf{t}\right)=K^{-1} B_{s-1}^{\star} S(0)$, then $K^{-1} B_{s-1}^{\star}=\frac{1}{S(0)}$. So that

$$
P\left(Y_{s}>v \mid \mathbf{t}\right)=\frac{S(v)}{S(0)}
$$

Notice that

$$
S(0)=\sum_{\ell=1}^{s} \frac{c_{\ell}}{\gamma_{\ell}} \int_{0}^{\infty} \int_{0}^{\infty} \eta \exp [-\delta] \mathrm{d} \beta_{1} \mathrm{~d} \beta_{2} .
$$

A $100 \tau \%$ BPIs for $Y_{s}, s=1, \ldots, m^{\star}$, can be obtained by solving the following two nonlinear equations for lower and upper bounds $L B$ and $U B$, respectively,

$$
\begin{gathered}
P\left(Y_{s}>L B \mid \mathbf{t}\right)=\frac{1+\tau}{2}, \\
P\left(Y_{s}>U B \mid \mathbf{t}\right)=\frac{1-\tau}{2},
\end{gathered}
$$

which are equivalent to the two equations

$$
\left.\begin{array}{r}
S(L B)-\left(\frac{1+\tau}{2}\right) S(0)=0, \\
S(U B)-\left(\frac{1-\tau}{2}\right) S(0)=0 .
\end{array}\right\}
$$

\section{APPLICATION TO A HALF-LOGISTIC DISTRIBUTION}

In this section, we discuss MLP and BPI for the s-th order statistics $Y_{s}$ under a half-logistic distribution. The half-logistic (or folded-logistic) distribution is one of the family of logistic distributions. It has been applied as a life testing model by Balakrishnan [24]. Many authors, including Balakrishnan and Puthenpura [25], Balakrishnan and Chan [26], Rosaiah et al. [27] and Kim and Han [28] have studied the halflogistic distribution.

Let $X$ be a logistic RV with CDF given by

$$
F_{x}(x)=(1+\exp [-\theta x])^{-1}, \quad-\infty<x<\infty, \quad(\theta>0)
$$

where $\theta$ is a scale parameter.

If $T=|X|$, and $\beta_{j} \equiv \theta$ then $T$ has a half-logistic distribution, the CDF for which is

$$
F_{T j}(t)=\frac{1-\exp \left[-\beta_{j} t\right]}{1+\exp \left[-\beta_{j} t\right]}, \quad t>0, \quad\left(\beta_{j}>0\right),
$$

and its PDF is given by

$$
f_{T j}(t)=\frac{2 \beta_{j} \exp \left[-\beta_{j} t\right]}{\left(1+\exp \left[-\beta_{j} t\right]\right)^{2}},
$$

which is monotone decreasing on $[0, \infty)$. Therefore, the PDF under competing risks model that corresponds to (5) takes the form

$$
f(t)=\left[\sum_{j=1}^{2} \frac{\beta_{j}}{1+\exp \left[-\beta_{j} t\right]}\right]\left[\prod_{j=1}^{2} \frac{2}{1+\exp \left[\beta_{j} t\right]}\right] .
$$

The PDFs given by Equations (22) and (23) and the HRFs are plotted in Figure 1. It can be noticed from this figure that the PDFs are all decreasing functions with mode at 0 , while the HRFs are increasing constant functions.

It may be noticed that Equation (21) can be obtained from (1) by putting

$$
u_{j}(t)=\ln \left[\frac{1+\exp \left[\beta_{j} t\right]}{2}\right]
$$

Using Equation (24), the MLP and BPI for $Y_{s}$ under a half-logistic distribution are given by solving (10) and (20), respectively. 

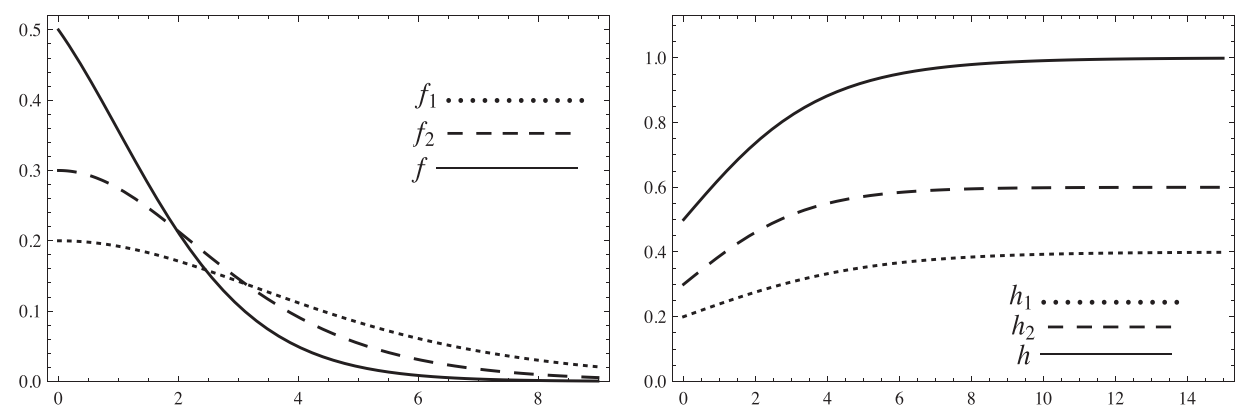

Figure 1 Left (Right) panel: The PDFs (HRFs) of the half-logistic distribution at $\beta_{1}=0.4$ and $\beta_{2}=0.6$ with their PDFs(HRFs) under competing risks model.

Remark 5.1. The half-logistic distribution includes one parameter which facilitates the calculations required in the estimation and prediction methods. It has also an advantage rather than the exponential distribution since the first has increasing hazard rate while the latter has constant hazard rate.

\section{SIMULATION STUDY AND ILLUSTRATIVE EXAMPLES}

\subsection{Simulation Study}

The following steps are followed to generate progressively type-II censored samples from CDF (21).

1. For given values of the prior parameters $\left(\alpha_{1}, \alpha_{2}, \alpha_{3}, \alpha_{4}\right)$, which are chosen to satisfy the following conditions of unbiased estimates,

$$
\begin{aligned}
& \mathrm{E}\left[B_{1}\right]=\frac{\alpha_{1}}{\alpha_{2}}=\beta_{1}, \\
& \mathrm{E}\left[B_{2}\right]=\frac{\alpha_{3}}{\alpha_{4}}=\beta_{2},
\end{aligned}
$$

where E denotes the expectation and $B_{1}$ and $B_{2}$ are two RVs with realizations $\beta_{1}$ and $\beta_{2}$, respectively, generate values for the parameters $\left(\beta_{1}, \beta_{2}\right)$, using Equations (12) and (13), see [29,30].

2. For given values of $n$ and $m(1<m<n)$, generate two independent random samples each of size $n$ from Uniform $(0,1)$ distribution, $\left(U_{1 j}, \cdots, U_{n j}\right), j=1,2$.

3. Generate two random samples $\left(t_{1 j}, \ldots, t_{n j}\right), j=1,2$, from CDF $(21)$ as follows

$$
t_{i j}=-\frac{1}{\beta_{j}} \ln \left[\frac{1-U_{i j}}{1+U_{i j}}\right], \quad i=1, \ldots, n, j=1,2 .
$$

4. Calculate $t_{i}$ such that, $t_{i}=\min \left\{t_{i 1}, t_{i 2}\right\}, i=1, \ldots, n$.

5. For given values of the CS $R_{1}, \ldots, R_{m}$ apply the progressive type-II censoring as described in Section 2, and hence obtain the ordered sample $\left(t_{1: m: n}, \ldots, t_{m: m: n}\right)$, which represents progressively type-II censored sample under competing risks model.

\subsection{Simulation Procedure}

In this subsection, a Monte Carlo simulation study is carried out in order to determine MLPs and BPIs for $Y_{s}, s=1, \ldots, m^{\star}$. The following six CSs are applied in the generation of the informative and future sample:

- CS1:

$$
\begin{aligned}
R_{i} & =1, & & i=1, \ldots, n-m, \\
R_{i} & =0, & & \text { otherwise, } \\
R_{i}^{\star} & =1, & & i=1, \ldots, n^{\star}-m^{\star}, \\
R_{i}^{\star} & =0, & & \text { otherwise, }
\end{aligned}
$$

which means that we remove one unit after each observed failure of the first $n-m\left(n^{\star}-m^{\star}\right)$ failures in the informative (future) sample. 
- CS2:

$$
\begin{aligned}
R_{i} & =n-m, & & i=1, \\
R_{i} & =0, & & \text { otherwise, } \\
R_{i}^{\star} & =n^{\star}-m^{\star}, & & i=1, \\
R_{i}^{\star} & =0, & & \text { otherwise, }
\end{aligned}
$$

which means that we remove $n-m\left(n^{\star}-m^{\star}\right)$ units after the first observed failure in the informative (future) sample.

- CS3:

$$
\begin{aligned}
R_{i} & =n-m, & & i=\frac{m}{2}, \\
R_{i} & =0, & & \text { otherwise, } \\
R_{i}^{\star} & =n^{\star}-m^{\star}, & & i=\frac{m^{\star}}{2}, \\
R_{i}^{\star} & =0, & & \text { otherwise, }
\end{aligned}
$$

which means that we remove $n-m\left(n^{\star}-m^{\star}\right)$ units after the middle observed failure in the informative (future) sample.

- CS4:

$$
\begin{aligned}
R_{i} & =n-m, & & i=m, \\
R_{i} & =0, & & \text { otherwise, } \\
R_{i}^{\star} & =n^{\star}-m^{\star}, & & i=m^{\star}, \\
R_{i}^{\star} & =0, & & \text { otherwise, }
\end{aligned}
$$

which means that we remove $n-m\left(n^{\star}-m^{\star}\right)$ units after the last observed failure in the informative (future) sample.

- CS5:

$$
\begin{aligned}
R_{i} & =\frac{n-m}{2}, & & i=1, m, \\
R_{i} & =0, & & \text { otherwise, } \\
R_{i}^{\star} & =\frac{n^{\star}-m^{\star}}{2}, & & i=1, m^{\star}, \\
R_{i}^{\star} & =0, & & \text { otherwise, }
\end{aligned}
$$

which means that we remove $\frac{n-m}{2}\left(\frac{n^{\star}-m^{\star}}{2}\right)$ units after the first and last observed failures in the informative (future) sample.

- CS6:

$$
\begin{aligned}
R_{i} & =\frac{n-m}{3}, & & i=1, \frac{m}{2}, m, \\
R_{i} & =0, & & \text { otherwise, } \\
R_{i}^{\star} & =\frac{n^{\star}-m^{\star}}{3}, & & i=1, \frac{m^{\star}}{2}, m^{\star}, \\
R_{i}^{\star} & =0, & & \text { otherwise, }
\end{aligned}
$$

which means that we remove $\frac{n-m}{3}\left(\frac{n^{\star}-m^{\star}}{3}\right)$ units after the first, middle and last observed failures in the informative (future) sample.

Through the simulation procedure, the values $m=18$ (with $n=30$ ), in the informative sample, and $m^{\star}=10$ (with $n^{\star}=10$ and 16 ), in the future sample, have been chosen.

Based on 1000 informative samples, MLPs for $Y_{s}, s=2,4,6,8,10$, biases and mean squared prediction errors (MSPEs) are presented in Table 1. A 95\% PIs for $Y_{s}, s=2,4,6,8,10$, in addition to the average interval lengths (AILs) of the PIs, are also included in Table 1.

The following steps are followed to determine the AILs and coverage probabilities (COVPs) of BPIs for $Y_{s}, s=2,4,6,8,10$. 
Table 1 MLPs for $Y_{s}$ with their Biases and MSPEs, in addition to 95\% PIs based on 1000 informative samples.

\begin{tabular}{|c|c|c|c|c|c|c|c|c|c|c|c|}
\hline \multirow[b]{2}{*}{ CS } & \multirow[b]{2}{*}{$Y_{s}$} & \multicolumn{4}{|c|}{$n^{\star}=m^{\star}=10$} & \multirow[b]{2}{*}{ AIL } & \multicolumn{5}{|c|}{$n^{\star}=16$ and $m^{\star}=10$} \\
\hline & & MLP & Bias & MSPE & PI & & MLP & Bias & MSPE & PI & AIL \\
\hline \multirow[t]{5}{*}{1} & $Y_{2}$ & 0.2074 & -0.1699 & 0.0952 & $(0.0938,0.3210)$ & 0.2272 & 0.1364 & -0.1200 & 0.0458 & $(0.0618,0.2109)$ & 0.1490 \\
\hline & $Y_{4}$ & 0.6553 & -0.1538 & 0.1655 & $(0.4202,0.8903)$ & 0.4701 & 0.4359 & -0.1159 & 0.0840 & $(0.2771,0.5947)$ & 0.3176 \\
\hline & $Y_{6}$ & 1.1984 & -0.1580 & 0.2800 & $(0.8206,1.5761)$ & 0.7555 & 0.8713 & -0.1391 & 0.1761 & $(0.5904,1.1523)$ & 0.5619 \\
\hline & $Y_{8}$ & 1.9042 & -0.2027 & 0.5185 & $(1.3437,2.4646)$ & 1.1209 & 1.5818 & -0.2235 & 0.4636 & $(1.1059,2.0576)$ & 0.9518 \\
\hline & $Y_{10}$ & 3.2687 & -0.5587 & 2.1717 & $(2.3248,4.2126)$ & 1.8878 & 2.9423 & -0.5790 & 2.1348 & $(2.0808,3.8038)$ & 1.7230 \\
\hline \multirow[t]{5}{*}{2} & $Y_{2}$ & 0.2044 & -0.1676 & 0.0928 & $(0.0938,0.3150)$ & 0.2212 & 0.1613 & -0.1524 & 0.0732 & $(0.0725,0.2501)$ & 0.1776 \\
\hline & $Y_{4}$ & 0.6535 & -0.1534 & 0.1651 & $(0.4223,0.8847)$ & 0.4624 & 0.5979 & -0.1582 & 0.1563 & $(0.3837,0.8122)$ & 0.4285 \\
\hline & $Y_{6}$ & 1.1902 & -0.1570 & 0.2759 & $(0.8219,1.5586)$ & 0.7367 & 1.1237 & -0.1610 & 0.2653 & $(0.7720,1.4755)$ & 0.7035 \\
\hline & $Y_{8}$ & 1.8977 & -0.2020 & 0.5142 & $(1.3472,2.4483)$ & 1.1010 & 1.8550 & -0.2074 & 0.5154 & $(1.3183,2.3917)$ & 1.0734 \\
\hline & $Y_{10}$ & 3.2552 & -0.5577 & 2.1585 & $(2.3404,4.1700)$ & 1.8296 & 3.2073 & -0.5602 & 2.1627 & $(2.3067,4.1080)$ & 1.8013 \\
\hline \multirow[t]{5}{*}{3} & $Y_{2}$ & 0.2059 & -0.1688 & 0.0941 & $(0.0924,0.3195)$ & 0.2270 & 0.1319 & -0.1160 & 0.0427 & $(0.0590,0.2047)$ & 0.1457 \\
\hline & $Y_{4}$ & 0.6555 & -0.1536 & 0.1656 & $(0.4204,0.8905)$ & 0.4701 & 0.3850 & -0.1008 & 0.0642 & $(0.2445,0.5255)$ & 0.2811 \\
\hline & $Y_{6}$ & 1.1930 & -0.1574 & 0.2769 & $(0.8148,1.5711)$ & 0.7563 & 0.7796 & -0.1549 & 0.1753 & $(0.5242,1.0351)$ & 0.5109 \\
\hline & $Y_{8}$ & 1.9166 & -0.2041 & 0.5254 & $(1.3502,2.4831)$ & 1.1329 & 1.5016 & -0.2393 & 0.4711 & $(1.0386,1.9647)$ & 0.9261 \\
\hline & $Y_{10}$ & 3.2943 & -0.5639 & 2.2038 & $(2.3432,4.2455)$ & 1.9023 & 2.9263 & -0.5896 & 2.2054 & $(2.0529,3.7997)$ & 1.7468 \\
\hline \multirow[t]{5}{*}{4} & $Y_{2}$ & 0.2071 & -0.1697 & 0.0956 & $(0.0909,0.3233)$ & 0.2324 & 0.1321 & -0.1161 & 0.0428 & $(0.0583,0.2059)$ & 0.1476 \\
\hline & $Y_{4}$ & 0.6611 & -0.1550 & 0.1682 & $(0.4184,0.9038)$ & 0.4854 & 0.3856 & -0.1010 & 0.0647 & $(0.2424,0.5288)$ & 0.2864 \\
\hline & $Y_{6}$ & 1.1850 & -0.1563 & 0.2741 & $(0.8050,1.5650)$ & 0.7600 & 0.6730 & -0.0965 & 0.0956 & $(0.4522,0.8938)$ & 0.4417 \\
\hline & $Y_{8}$ & 1.9130 & -0.2038 & 0.5244 & $(1.3457,2.4804)$ & 1.1347 & 1.0037 & -0.0970 & 0.1375 & $(0.6959,1.3114)$ & 0.6156 \\
\hline & $Y_{10}$ & 3.2265 & -0.5529 & 2.1219 & $(2.2794,4.1737)$ & 1.8942 & 1.3496 & -0.1011 & 0.1855 & $(0.9511,1.7481)$ & 0.7970 \\
\hline \multirow[t]{5}{*}{5} & $Y_{2}$ & 0.2070 & -0.1696 & 0.0948 & $(0.0926,0.3213)$ & 0.2287 & 0.1436 & -0.1278 & 0.0521 & $(0.0643,0.2230)$ & 0.1587 \\
\hline & $Y_{4}$ & 0.6579 & -0.1542 & 0.1664 & $(0.4218,0.8940)$ & 0.4722 & 0.4681 & -0.1219 & 0.0942 & $(0.2974,0.6387)$ & 0.3412 \\
\hline & $Y_{6}$ & 1.1942 & -0.1574 & 0.2775 & $(0.8182,1.5703)$ & 0.7520 & 0.8334 & -0.1180 & 0.1447 & $(0.5684,1.0984)$ & 0.5300 \\
\hline & $Y_{8}$ & 1.9129 & -0.2037 & 0.5227 & $(1.3540,2.4718)$ & 1.1178 & 1.2810 & -0.1255 & 0.2246 & $(0.9010,1.6610)$ & 0.7600 \\
\hline & $Y_{10}$ & 3.2281 & -0.5528 & 2.1292 & $(2.2968,4.1594)$ & 1.8626 & 1.8263 & -0.1527 & 0.3604 & $(1.2994,2.3532)$ & 1.0539 \\
\hline \multirow[t]{5}{*}{6} & $Y_{2}$ & 0.2079 & -0.1704 & 0.0957 & $(0.0927,0.3266)$ & 0.2302 & 0.1386 & -0.1224 & 0.0478 & $(0.0618,0.2153)$ & 0.1535 \\
\hline & $Y_{4}$ & 0.6567 & -0.1539 & 0.1663 & $(0.4208,0.8931)$ & 0.4728 & 0.4335 & -0.1130 & 0.0811 & $(0.2748,0.5922)$ & 0.3174 \\
\hline & $Y_{6}$ & 1.1750 & -0.1549 & 0.2692 & $(0.8049,1.5451)$ & 0.7402 & 0.8020 & -0.1210 & 0.1418 & $(0.5403,1.0636)$ & 0.5232 \\
\hline & $Y_{8}$ & 1.9221 & -0.2047 & 0.5271 & $(1.3592,2.4850)$ & 1.1258 & 1.3202 & -0.1461 & 0.2625 & $(0.9218,1.7185)$ & 0.7967 \\
\hline & $Y_{10}$ & 3.2717 & -0.5605 & 2.1771 & $(2.3342,4.2091)$ & 1.8749 & 2.0127 & -0.2023 & 0.5063 & $(1.4302,2.5952)$ & 1.1651 \\
\hline
\end{tabular}

1. Generate a progressively type-II censored random sample under competing risks model as shown in Subsection 6.1. This sample will be called "informative sample." Based on this sample, calculate BPIs for $Y_{s}, s=2,4,6,8,10$.

2. Generate another sample (will be called "future sample") and assign the values of $Y_{s}, s=2,4,6,8,10$, and then determine whether the values of $Y_{s}, s=2,4,6,8,10$ lie in their corresponding BPIs or not.

3. Repeat the above two steps 1000 times to induce $1000 \mathrm{BPIs}$ for $Y_{s}, s=2,4,6,8,10$.

4. Calculate the average of the BPIs and hence calculate the AILs of the BPIs.

5. Calculate the COVPs of the BPIs as follows

$$
\text { COVP }=\frac{\text { Number of BPIs that include } Y_{s}}{1000} .
$$

The prior parameter values $\alpha_{1}=0.30, \alpha_{2}=0.75, \alpha_{3}=0.57$ and $\alpha_{4}=0.95$ have been taken so as to generate $\beta_{1}=0.4$ and $\beta_{2}=0.6$, using (12) and (13). The 95\% BPIs for $Y_{s}, s=2,4,6,8,10$, AILs and COVPs of the BPIs are presented in Table 2.

\subsection{Illustrative Examples}

\subsubsection{Example 1}

Based on the values of $\beta_{1}$ and $\beta_{2}$, generated above, generate two independent random samples each of which of size $n=30$ from CDF (21) and then apply step 4 in Subsection 6.1 to them in order to obtain a random sample of size $n=30$ under competing risks model. Apply CS1 to this sample to obtain a progressively type-II censored sample of size $m=18$. This sample is called the informative sample. 
Table 2 The AILs and COVPs of 95\% BPIs for $Y_{s}$. Prior parameter values: $\alpha_{1}=0.30, \alpha_{2}=0.75$, $\alpha_{3}=0.57$ and $\alpha_{4}=0.95$. Population parameter values: $\beta_{1}=0.4$ and $\beta_{2}=0.6$.

\begin{tabular}{|c|c|c|c|c|c|c|c|}
\hline \multirow[b]{2}{*}{ CS } & \multirow[b]{2}{*}{$Y_{s}$} & \multicolumn{3}{|c|}{$n^{\star}=m^{\star}=10$} & \multicolumn{3}{|c|}{$n^{\star}=16$ and $n^{\star}=10$} \\
\hline & & BPI & AIL & COVP & BPI & AIL & COVP \\
\hline \multirow[t]{5}{*}{1} & $Y_{2}$ & $(0.0503,1.1472)$ & 1.0969 & 95.4 & $(0.3021,0.7611)$ & 0.7290 & 96.1 \\
\hline & $Y_{4}$ & $(0.2460,1.9967)$ & 1.7507 & 95.4 & $(0.1660,1.4125)$ & 1.2465 & 94.8 \\
\hline & $Y_{6}$ & $(0.5349,2.9466)$ & 2.4118 & 94.2 & $(0.3939,2.3073)$ & 1.9134 & 95.2 \\
\hline & $Y_{8}$ & $(0.9552,4.3893)$ & 3.4341 & 95.7 & $(0.7864,3.9453)$ & 3.1589 & 95.4 \\
\hline & $Y_{10}$ & $(1.7136,8.2085)$ & 6.4948 & 95.1 & $(1.5304,7.9319)$ & 6.4014 & 96.1 \\
\hline \multirow[t]{5}{*}{2} & $Y_{2}$ & $(0.0503,1.1438)$ & 1.0936 & 95.6 & $(0.0403,0.9910)$ & 0.9507 & 95.9 \\
\hline & $Y_{4}$ & $(0.2444,1.9728)$ & 1.7283 & 95.7 & $(0.2193,1.8554)$ & 1.6360 & 94.9 \\
\hline & $Y_{6}$ & $(0.5383,2.9472)$ & 2.4089 & 94.0 & $(0.5097,2.8744)$ & 2.3647 & 95.4 \\
\hline & $Y_{8}$ & $(0.9607,4.3864)$ & 3.4257 & 94.8 & $(0.9236,4.3003)$ & 3.3767 & 96.6 \\
\hline & $Y_{10}$ & $(1.7299,8.2343)$ & 6.5044 & 95.9 & $(1.6826,8.1171)$ & 6.4344 & 95.0 \\
\hline \multirow[t]{5}{*}{3} & $Y_{2}$ & $(0.0506,1.1567)$ & 1.1061 & 96.0 & $(0.0313,0.7442)$ & 0.7128 & 96.4 \\
\hline & $Y_{4}$ & $(0.2454,1.9937)$ & 1.7483 & 94.8 & $(0.1501,1.2732)$ & 1.1231 & 95.0 \\
\hline & $Y_{6}$ & $(0.5434,2.9973)$ & 2.4540 & 94.8 & $(0.3460,2.1649)$ & 1.8189 & 94.9 \\
\hline & $Y_{8}$ & $(0.9490,4.3704)$ & 3.4214 & 96.3 & $(0.7366,3.8634)$ & 3.1268 & 93.4 \\
\hline & $Y_{10}$ & $(1.7089,8.1976)$ & 6.4887 & 94.7 & $(1.4874,7.9135)$ & 6.4262 & 95.6 \\
\hline \multirow[t]{5}{*}{4} & $Y_{2}$ & $(0.0506,1.1604)$ & 1.1098 & 95.9 & $(0.0307,0.7315)$ & 0.7008 & 94.1 \\
\hline & $Y_{4}$ & $(0.2430,1.9829)$ & 1.7399 & 95.5 & $(0.1472,1.2538)$ & 1.1066 & 95.8 \\
\hline & $Y_{6}$ & $(0.5422,3.0050)$ & 2.4628 & 94.8 & $(0.3051,1.7518)$ & 1.4467 & 95.4 \\
\hline & $Y_{8}$ & $(0.9418,4.3597)$ & 3.4179 & 95.0 & $(0.5059,2.3433)$ & 1.8374 & 95.6 \\
\hline & $Y_{10}$ & $(1.7081,8.2391)$ & 6.5310 & 96.2 & $(0.7673,3.1182)$ & 2.3509 & 96.2 \\
\hline \multirow[t]{5}{*}{5} & $Y_{2}$ & $(0.0510,1.1638)$ & 1.1128 & 95.6 & $(0.0349,0.8324)$ & 0.7975 & 94.9 \\
\hline & $Y_{4}$ & $(0.2445,1.9835)$ & 1.7390 & 95.0 & $(0.1774,1.4987)$ & 1.3213 & 96.7 \\
\hline & $Y_{6}$ & $(0.5405,2.9770)$ & 2.4365 & 93.9 & $(0.3800,2.1550)$ & 1.7750 & 95.9 \\
\hline & $Y_{8}$ & $(0.9524,4.3757)$ & 3.4233 & 95.6 & $(0.6366,2.9208)$ & 2.2842 & 95.7 \\
\hline & $Y_{10}$ & $(1.7248,8.2572)$ & 6.5323 & 95.2 & $(1.0010,4.0860)$ & 3.0850 & 95.7 \\
\hline \multirow[t]{5}{*}{6} & $Y_{2}$ & $(0.0511,1.1665)$ & 1.1154 & 94.0 & $(0.0335,0.7978)$ & 0.7642 & 95.4 \\
\hline & $Y_{4}$ & $(0.2428,1.9702)$ & 1.7273 & 94.5 & $(0.1629,1.3767)$ & 1.2139 & 94.7 \\
\hline & $Y_{6}$ & $(0.5403,2.9783)$ & 2.4380 & 95.2 & $(0.3668,2.1147)$ & 1.7479 & 95.9 \\
\hline & $Y_{8}$ & $(0.9589,4.4068)$ & 3.4479 & 94.6 & $(0.6592,3.1088)$ & 2.4496 & 95.7 \\
\hline & $Y_{10}$ & $(1.7203,8.2415)$ & 6.5212 & 95.3 & $(1.0867,4.6203)$ & 3.5335 & 96.4 \\
\hline
\end{tabular}

Assume that (i) $\left(n^{\star}, m^{\star}\right)=(10,10)$ (no censoring) and (ii) $\left(n^{\star}, m^{\star}\right)=(16,10)$ (six values are censored). Based on CS1 we generate another progressively type-II censored sample under competing risks model, using CDF (21), as before. This sample is called the future sample. Table 3 displays, in the second column, the original random sample of size 30 generated from CDF (21) due to the first cause of failure $\left(\beta_{1}=0.4\right)$, while that due to the second cause of failure $\left(\beta_{2}=0.6\right)$ is presented in the third column. The values of these two samples are then compared and the minimum values are recorded in the fourth column. The values of progressively type-II censored samples of sizes 18 are presented in the fifth columns. The sample in the last column is called informative sample. Table 4 shows some selected values of the future order statistics which will be proposed to determine their MLPs and BPIs, while Table 5 shows MLPs and 95\% BPIs for the selected values in Table 4. The PMLEs of $\beta_{1}$ and $\beta_{2}$ are $\widehat{\beta_{1}}=0.3048$ and $\widehat{\beta_{2}}=0.6950$ when $n^{\star}=10$ and $\widehat{\beta_{1}}=0.3049$ and $\widehat{\beta_{2}}=0.6949$ when $n^{\star}=16$.

\subsubsection{Example 2}

Our object, in this example, is to predict with the $s$-th order statistic, $Y_{s}$, in the future sample, $s=1, \ldots, m^{\star}$, based on a data set originally analyzed by Hoel [31]. The data were obtained from a laboratory experiment in which 77 male mice exposed to a radiation dose of 300 roentgens at 5 to 6 weeks of age. The cause of death for each mouse was determined by autopsy to be thymine lymphoma, reticulum cell sarcoma or other causes. For the purpose of analysis, we restrict the causes of death to two causes only considering reticulum cell sarcoma as cause 1 and combining the other causes of death as cause 2. Kundu et al. [32] generated a progressively type-II censored sample from the original data with $m=25$. The CS is given by $R_{i}=2, i=1, \ldots, 24$ and $R_{25}=4$. The progressively type-II censored sample used by Kundu et al. [32], Pareek et al. [33] and Cramer and Schmiedt [18] is given by 
Table 3 Informative progressively type-II censored samples. Prior parameter values: $\alpha_{1}=0.30, \alpha_{2}=0.75, \alpha_{3}=0.57$ and $\alpha_{4}=0.95$. Population parameter values: $\beta_{1}=0.4$ and $\beta_{2}=0.6$.

\begin{tabular}{|c|c|c|c|c|}
\hline$i$ & $T_{i 1}$ & $T_{i 2}$ & $T_{i}$ & $T_{i ; 18 ; 30}$ \\
\hline 1 & 5.59979 & 5.76755 & 5.59979 & 0.01603 \\
\hline 2 & 1.73549 & 1.63035 & 1.63035 & 0.15559 \\
\hline 3 & 1.38032 & 2.71452 & 1.38032 & 0.18830 \\
\hline 4 & 1.48707 & 2.01749 & 1.48707 & 0.31341 \\
\hline 5 & 8.95048 & 3.23940 & 3.23940 & 0.34363 \\
\hline 6 & 0.48371 & 9.14179 & 0.48371 & 0.41885 \\
\hline 7 & 0.79873 & 1.35164 & 0.79873 & 0.46795 \\
\hline 8 & 5.42455 & 0.57271 & 0.57271 & 0.57271 \\
\hline 9 & 4.07025 & 2.72627 & 2.72627 & 0.70169 \\
\hline 10 & 4.22138 & 3.51768 & 3.51768 & 0.72990 \\
\hline 11 & 2.89722 & 0.15559 & 0.15559 & 0.85833 \\
\hline 12 & 8.68340 & 0.31341 & 0.31341 & 1.00249 \\
\hline 13 & 3.77322 & 3.73408 & 3.73408 & 1.82999 \\
\hline 14 & 1.53726 & 3.16222 & 1.53726 & 2.21175 \\
\hline 15 & 3.17939 & 1.43613 & 1.43613 & 2.22394 \\
\hline 16 & 0.85833 & 1.15286 & 0.85833 & 2.36294 \\
\hline 17 & 5.37478 & 0.41885 & 0.41885 & 3.01668 \\
\hline 18 & 7.13561 & 2.22394 & 2.22394 & 6.14015 \\
\hline 19 & 4.79655 & 1.00249 & 1.00249 & \\
\hline 20 & 4.20190 & 0.70169 & 0.70169 & \\
\hline 21 & 4.63921 & 2.21175 & 2.21175 & \\
\hline 22 & 0.46795 & 1.70203 & 0.46795 & \\
\hline 23 & 2.93812 & 2.36294 & 2.36294 & \\
\hline 24 & 0.34363 & 1.15989 & 0.34363 & \\
\hline 25 & 1.54094 & 0.01603 & 0.01603 & \\
\hline 26 & 6.14015 & 6.22725 & 6.14015 & \\
\hline 27 & 1.82999 & 5.68222 & 1.82999 & \\
\hline 28 & 1.42938 & 0.72990 & 0.72990 & \\
\hline 29 & 3.74519 & 3.01668 & 3.01668 & \\
\hline 30 & 5.85944 & 0.18830 & 0.18830 & . \\
\hline
\end{tabular}

Table 4 Selected values for $Y_{s}$.

\begin{tabular}{ccccccc}
\hline & & \multicolumn{6}{c}{$\boldsymbol{Y s}$} \\
\cline { 3 - 7 } $\boldsymbol{n}^{*}$ & $\boldsymbol{m}^{*}$ & $\boldsymbol{Y}_{\mathbf{2}}$ & $\boldsymbol{Y}_{\mathbf{4}}$ & $\boldsymbol{Y}_{\mathbf{6}}$ & $\boldsymbol{Y}_{\mathbf{8}}$ & $\boldsymbol{Y}_{\mathbf{1 0}}$ \\
\hline 10 & 10 & 0.0520 & 0.8016 & 0.9920 & 1.5103 & 3.7583 \\
16 & 10 & 0.0520 & 0.6533 & 0.8259 & 1.1179 & 5.8339 \\
\hline
\end{tabular}

Table 5 MLPs and 95\% BPIs for the values of $Y_{s}$ that given in Table 4.

\begin{tabular}{|c|c|c|c|c|}
\hline \multirow[b]{2}{*}{$Y_{s}$} & \multicolumn{2}{|c|}{$n^{\star}=m^{\star}=10$} & \multicolumn{2}{|c|}{$n^{\star}=16$ and $m^{\star}=10$} \\
\hline & MLP & BPI & MLP & BPI \\
\hline$Y_{2}$ & 0.2160 & $(0.0525,1.1930)$ & 0.1358 & $(0.0334,0.7895)$ \\
\hline$Y_{4}$ & 0.6818 & $(0.2527,2.0421)$ & 0.4572 & $(0.1687,1.4314)$ \\
\hline$Y_{6}$ & 1.2314 & $(0.5576,3.0651)$ & 0.9021 & $(0.4029,2.3538)$ \\
\hline$Y_{8}$ & 1.9692 & $(0.9986,4.5919)$ & 1.6373 & $(0.8119,4.0709)$ \\
\hline$Y_{10}$ & 3.3635 & $(1.8121,8.7346)$ & 3.0640 & $(1.6033,8.3531)$ \\
\hline
\end{tabular}


$(252,2,2),(259,2,2),(318,1,2),(385,2,2),(407,2,2),(420,2,2),(462,2,2),(517,2,2),(517,2,2),(524,2,2),(525,1,2),(536,1,2),(558$, $1,2),(605,1,2),(612,1,2),(620,2,2),(621,1,4)$,

where the first component represents the lifetime, the second component indicates the cause of death and the third component denotes the censoring value.

The lifetimes of the mice are assumed to be independent and subject to the exponential distribution in Kundu et al. [32], Weibull distribution in Pareek et al. [33] and Lomax distribution in Cramer and Schmiedt [18].

It can be easily shown that the above progressively type-II censored data could come from CDF (3) with $\mathfrak{u}_{j}(t)$, given by (24), using Kolmogorov-Smirnov test. The empirical CDF used in this test may be written as, see [14],

$$
\tilde{F}\left(t_{i}: m: n\right)=1-\prod_{j=1}^{i}\left(1-\tilde{p}_{j}\right), \quad i=1, \ldots, m,
$$

where

$$
\tilde{p}_{j}=\frac{1}{n-\left[\sum_{k=2}^{j} R_{k-1}\right]-j+1}, \quad j=1, \ldots, m .
$$

From the above data and using $\operatorname{CDF}(3)$ with $u_{j}(t)$, given by (24), it can be shown that the maximum likelihood estimates (MLEs) of $\beta_{1}$ and $\beta_{2}$ are given, respectively, by $\widetilde{\beta_{1}}=0.000481$ and $\widetilde{\beta_{2}}=0.001116$. The Kolmogorov-Smirnov test statistic $=0.22687$ and the P-value $=0.99982$. Therefore, there is a good possibility for the given data to be come from CDF (3) with $u_{j}(t)$, given by $(24)$. Under the above progressively type-II censored data, the logarithm of likelihood Equation (6) with $u_{j}(t)$, given by (24) is plotted in Figure 2 . It can be noticed from this figure that the plane attains its maximum at $\widetilde{\beta_{1}}=0.000481$ and $\widetilde{\beta_{2}}=0.001116$.

The prior parameter values have been taken to be $\alpha_{1}=0.0030, \alpha_{2}=2.9000, \alpha_{3}=0.0071$ and $\alpha_{4}=5.1000$ to generate population parameter values $\widetilde{\beta_{1}}=0.000481$ and $\widetilde{\beta_{2}}=0.001116$ using (12) and (13). Suppose that $m^{\star}=10$ (with $n^{\star}=10$ and $n^{\star}=16$ later on) and applying CS4. Table 6 shows MLPs for $Y_{s}$, in the future sample, $s=2,4,6,8,10$. The PMLEs of $\beta_{1}$ and $\beta_{2}$ are $\widehat{\beta_{1}}=0.0 .000497$ and $\widehat{\beta_{2}}=0.001152$ when $n^{\star}=10$ and 16. Table 7 shows $95 \%$ BPIs for $Y_{s}$, in the future sample, $s=2,4,6,8,10$. For 100,000 generated future ordered samples each of which of size $m^{\star}=10$ under competing risks model using CDF (3) with $u_{j}(t)$, given by (24), considering the parameters $\widetilde{\beta_{1}}=0.000481$ and $\widetilde{\beta_{2}}=0.001116$. The COVPs of the BPIs for $Y_{s}, s=2,4,6,8,10$ have been also calculated and presented in Table 7 .

\section{CONCLUDING REMARKS}

In this paper, under competing risks model and based on a progressively type-II censored informative sample generated from the half-logistic distribution, we have discussed MLPs and BPIs for future progressively type-II censored competing risks data from the same population.

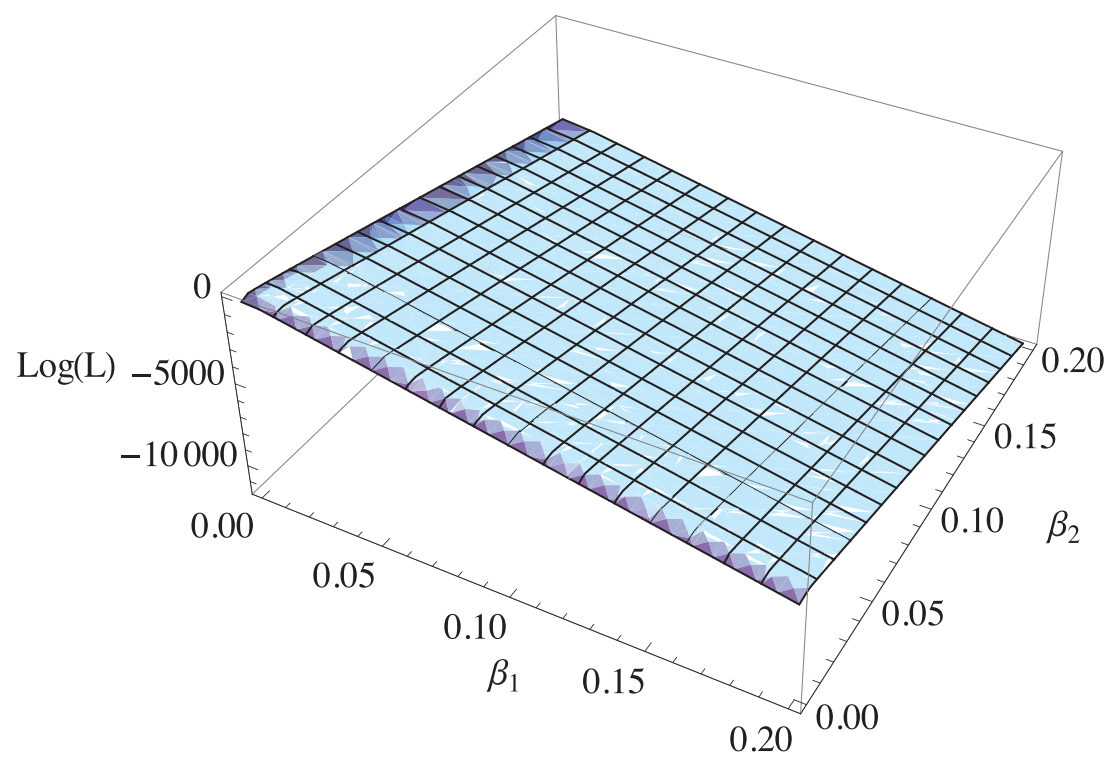

Figure 2 The logarithm of $\mathrm{L}\left(\beta_{1}, \beta_{2}\right)$ under progressively type-II censored competing risks data. 
Table 6 MLPs for $Y_{s}$. Prior parameter values: $\alpha_{1}=0.0030, \alpha_{2}=2.9000, \alpha_{3}=0.0071$ and $\alpha_{4}=5.1000$. Population parameter values: $\widetilde{\beta}_{1}=0.000481$ and $\widetilde{\beta}_{2}=0.001116$.

\begin{tabular}{rrrrrrr}
\hline & & \multicolumn{6}{c}{$\boldsymbol{Y s}$} \\
\cline { 3 - 7 } $\boldsymbol{n}^{*}$ & $\boldsymbol{m}^{*}$ & \multicolumn{1}{c}{$\boldsymbol{Y}_{\mathbf{2}}$} & $\boldsymbol{Y}_{\mathbf{4}}$ & \multicolumn{1}{c}{$\boldsymbol{Y}_{\mathbf{6}}$} & \multicolumn{1}{c}{$\boldsymbol{Y}_{\mathbf{8}}$} & \multicolumn{1}{c}{$\boldsymbol{Y}_{\mathbf{1 0}}$} \\
\hline 10 & 10 & 130.9690 & 413.3470 & 746.4020 & 1193.3700 & 2037.9600 \\
16 & 10 & 79.5712 & 245.7490 & 425.3320 & 625.3520 & 857.8680 \\
\hline
\end{tabular}

Table 7 The COVPs in \% and 95\% BPIs for $Y_{s}$.

\begin{tabular}{lccccc}
\hline & \multicolumn{2}{c}{$\boldsymbol{n}^{*}=\boldsymbol{m}^{*}=\mathbf{1 0}$} & & & \multicolumn{2}{c}{$\boldsymbol{n}^{*}=\mathbf{1 6}$ and $\boldsymbol{m}^{*}=\mathbf{1 0}$} \\
\cline { 2 - 3 } \cline { 5 - 6 } $\boldsymbol{Y}_{\boldsymbol{s}}$ & $\mathbf{2 P I}$ & COVP & & BPI & COVP \\
\hline$Y_{2}$ & $(31.6553,703.4732)$ & 96.0 & & $(19.4418,449.9195)$ & 95.9 \\
$Y_{4}$ & $(153.0676,1197.9496)$ & 96.7 & & $(90.4819,740.9591)$ & 96.7 \\
$Y_{6}$ & $(338.9109,1792.2874)$ & 97.1 & & $(190.2302,1042.1497)$ & 97.1 \\
$Y_{8}$ & $(608.4114,2681.1188)$ & 97.5 & & $(313.3242,1378.8600)$ & 97.5 \\
$Y_{10}$ & $(1105.0914,5116.6316)$ & 97.2 & & $(463.0192,1781.5689)$ & 97.9 \\
\hline
\end{tabular}

An illustrative example based on simulated data and another one based on real data have been considered to investigate the performance of prediction.

From Table 1 we observe the following:

1. For all considered CSs, by increasing the index $s$ the MSPEs and AILs increase.

2. In the fourth column in which we have considered the progressive censoring in future sample, the MSPEs and AILs give less values with respect to the last three CSs than those for the above CSs (CS1, CS2, CS3).

From Table 2 we observe the following:

1. The COVPs of the BPIs are quite close to the nominal confidence levels $95 \%$.

2. For all considered CSs, by increasing the index $s$ the AILs increase.

3. In the fourth column in which we have considered the progressive censoring in future sample, better results for the BPIs, through smaller values for the AILs and closer values for the COVPs (near to 95\%) have been obtained. This is more clear in the last three CSs.

4. CS4, in which we remove all the surviving units after the last failure, gives better results with respect to the AILs, than the other CSs.

5. Numerical computations show that the above results do not change by changing the values of the prior parameters.

6. If the hyperparameters are unknown, "simple empirical Bayes estimators" may be obtained by using "past samples," see [34]. Alternatively, one could use hierarchical Bayes approach in which a suitable prior for the vector of hyperparameters is used, see [35].

\section{CONFLICT OF INTEREST}

Essam. K. AL-Hussaini contributes with the review of the paper. Alaa H. Abdel-Hamid contributes with the idea and preparation of the paper. Atef. F. Hashem contributes with derivation (calculation) of the analytical (numerical) results in addition to writing the paper.

\section{REFERENCES}

1. E.K. AL-Hussaini, J. Stat. Plan. Inf. 79 (1999), 79-91.

2. E.K. AL-Hussaini, J. Stat. Plan. Inf. 113 (2003), 15-24.

3. M.A.M. Ali Mousa, J. Stat. Comput. Simul. 71 (2001), 163-181.

4. Z.F. Jaheen, Commun. Stat. Simul. Comput. 32 (2003), 663-676.

5. I. Basak, P. Basak, N. Balakrishnan, Comput. Stat. Data Anal. 50 (2006), 1313-1337.

6. I. Basak, N. Balakrishnan, Sankhyā. 71 (2009), 222-247.

7. M.Z. Raqab, A. Asgharzadeh, R. Valiollahi, Comput. Stat. Data Anal. 54 (2010), 1732-1743. 
8. M.M. Mohie El-Din, A. Shafay, Stat. Pap. 54 (2013), 287-307.

9. A.H. Abdel-Hamid, E.K. AL-Hussaini, J. Stat. Comput. Simul. 84 (2014), 1297-1312.

10. E.K. AL-Hussaini, A.H. Abdel-Hamid, A.F. Hashem, J. Egypt. Math. Soc. 23 (2015), 190-196.

11. E.K. AL-Hussaini, M.I. Osman, J. Stat. Comput. Simul. 58 (1997), 121-144.

12. A.H. Abdel-Hamid, E.K. AL-Hussaini, Metrika. 66 (2007), 213-231.

13. N.R. Mann, R.E. Schafer, N.D. Singpurwalla, Methods for Statistical Analysis of Reliability and Life Data, Wiley, New York, NY, USA, 1974.

14. W.Q. Meeker, L.A. Escobar, Statistical Methods for Reliability Data, Wiley, New York, NY, USA, 1998.

15. J.F. Lawless, Statistical Models and Methods for Lifetime Data, second ed., Wiley, New York, NY, USA, 2003.

16. N. Balakrishnan, R. Aggarwala, Progressive Censoring: Theory, Methods and Applications, Birkhauser Publishers, Boston, MA, USA, 2000.

17. N. Balakrishnan, E. Cramer, The Art of Progressive Censoring: Applications to Reliability and Quality, Birkhäuser, New York, NY, USA, 2014.

18. E. Cramer, A.B. Schmiedt, Comput. Stat. Data Anal. 55 (2011), 1285-1303.

19. N. Balakrishnan, E. Cramer, U. Kamps, Stat. Probab. Lett. 54 (2001), 301-315.

20. U. Kamps, E. Cramer, Statistics. 35 (2001), 269-280.

21. W. Nelson, Accelerated Testing: Statistical Models, Test Plans and Data Analysis, Wiley, New York, NY, USA, 1990.

22. A.H. Abdel-Hamid, E.K. AL-Hussaini, J. Comput. Appl. Math. 235 (2011), 5259-5271.

23. R.A. Waller, M.S. Waterman, SIAM Rev. 20 (1978), 820-856.

24. N. Balakrishnan, J. Stat. Comput. Simul. 20 (1985), 287-309.

25. N. Balakrishnan, S. Puthenpura, J. Stat. Comput. Simul. 25 (1986), 193-204.

26. N. Balakrishnan, P.S. Chan, Comput. Stat. Data Anal. 13 (1992), 123-141.

27. K. Rosaiah, R.R.L. Kantam, B. Srinivasa Rao, Calcutta Stat. Assoc. Bull. 61 (2009), 183-196.

28. C. Kim, K. Han, Stat. Pap. 51 (2010), 375-387.

29. E.K. AL-Hussaini, A.H. Abdel-Hamid, Commun. Stat. Simul. Comput. 33 (2004), 963-982.

30. A.H. Abdel-Hamid, J. Egypt. Math. Soc. 16 (2008), 75-98.

31. D.G. Hoel, Biometrics. 28 (1972), 475-488.

32. D. Kundu, N. Kannan, N. Balakrishnan, in: N. Balakrishnan, C.R. Rao (Eds.), Handbook of Statistics, vol. 23, Elsevier, Amsterdam, Netherlands, 2004, pp. 331-348.

33. B. Pareek, D. Kundu, S. Kumar, Comput. Stat. Data Anal. 53 (2009), 4083-4094.

34. J. Maritz, T. Lwin, Empirical Bayes Methods, second ed., Chapman and Hall, London, England, 1989.

35. J.M. Bernardo, A.F.M. Smith, Bayesian Theory, Wiley, New York, NY, USA, 1994. 